\title{
Use of track-before-detect algorithm to reduce settling period of Kalman filter
}

M. Okoń-Fąfara, B. Wajszczyk

M. Okoń-Fąfara, B. Wajszczyk, "Use of track-before-detect algorithm to reduce settling period of Kalman filter," Proc. SPIE 11442, Radioelectronic Systems Conference 2019, 114421B (11 February 2020); doi: $10.1117 / 12.2565754$

SPIE. Event: Radioelectronic Systems Conference 2019, 2019, Jachranka, Poland 


\title{
Use of Track-Before-Detect algorithm to reduce settling period of Kalman filter
}

\author{
M. Okoń-Fąfara*, B. Wajszczyk \\ Faculty of Electronics, Military University of Technology, \\ Kaliskiego Str. 2, 00-908 Warsaw
}

\begin{abstract}
A settling time of the Kalman filter is a common problem of track initiation. The achievement of a steady state takes a few periods between consecutive measurements. In the case of the ABT objects (Air Breathing Target) the settling problem is negligible but should be considered for targets with high linear velocity like space crafts or tactical ballistic missiles. It is caused by a big distance between consecutive position measurements and the non-linearity of object's trajectory. To reduce the settling period the track formation using Track-Before-Detect (TBD) algorithms can be used. The TBD methods allows to preselect measurements collected during few scanning periods and its filtering should reduce the time of duration of the Kalman filter's unsteady state. In this paper a comparison of tracking results of classical tracking by Kalman filter and with pre-processing using chosen TBD algorithm is presented.
\end{abstract}

Keywords: tactical ballistic missile, tracking, Track-Before-Detect, computational optimization

\section{INTRODUCTION}

Multiple target tracking is one of the basic functions of the majority of modern surveillance radars. Many methods and tracking algorithms are based on the Kalman filter (KF), which is one of the suboptimal filtering method [1]. The tracking module using the KF can be divided into steps presented in Figure 1.

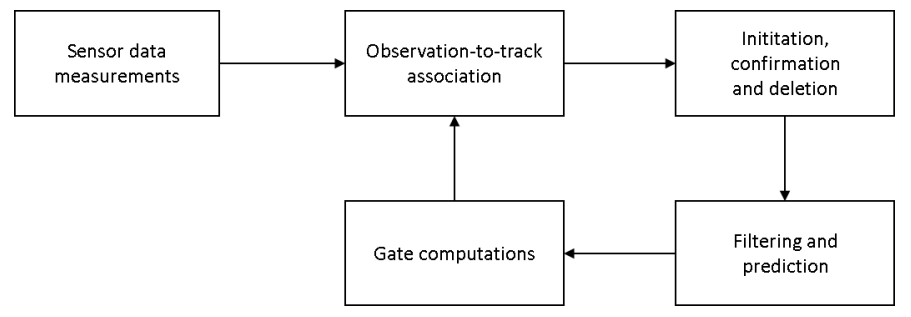

Figure 1. Tracking module scheme [1].

Every data received from a sensor should be considered for the update of existing tracks. The negative association decision gives an opportunity to initiate a new track. The stage of initiation is very significant, because the KF reaches it settled state after some number of algorithm's iteration depended on a scanning period, an assumed initial conditions and an object's dynamics [2].

In the case of the class of the objects called Air Breathing Targets, that includes aircrafts, the object's dynamics is relatively small what ensures short filter's settling time. Much more challenging are fast moving objects, which can dislocate by few sensor resolution from scan to scan. Such behavior characterizes the space crafts or tactical ballistic missiles (TBM). In such case the track initiation requires very wide correlations gates, which can include many plots. The gate narrowing could lead to quick track deletion.

To avoid problems with fast moving targets, an alternative approach called Track-Before-Detect (TBD) should be considered. In such case plot data are collected by few consecutive scans and if the chosen set of plots meets the decision function requirements, the detection is confirmed [1]. Then, the detection result can be transmitted for further tracking

\footnotetext{
*marta.okon@wat.edu.pl; phone +48 261839 243; fax +48 261837 461; wel.wat.edu.pl 
[3]. In effect, the TBD algorithm can be treated as initiation step of Kalman filter. The aim of this paper is to examine this statement by the use of the dedicated TBM tracking chain.

\section{TRACKING CHAIN}

Implementation of tracking chain is based on the TBD algorithms designed to detect tactical ballistic missiles. In the set of data collected by $N$ scans, it is expected to occur at least $M$ candidate plots, which fit a template. The templates consist of the parametrized correlation gates and they are stored in the database. Every time a plot is in the chosen correlation gate, the detection flag is set to one, otherwise it is set to zero. Therefore, this algorithm is commonly known as M/N or binary integrator [3].

Templates design should consider the distance between sensor and object, predicted course of object's trajectory, launch angle, antenna time of revolution, and the lack of correlation between the launch time and the antenna. An example of such prepared template is represented by the black squares in figure 2, where the first plot is marked by the yellow point, the red ones are consecutively detected plots of the object's trajectory drawn with the blue line.

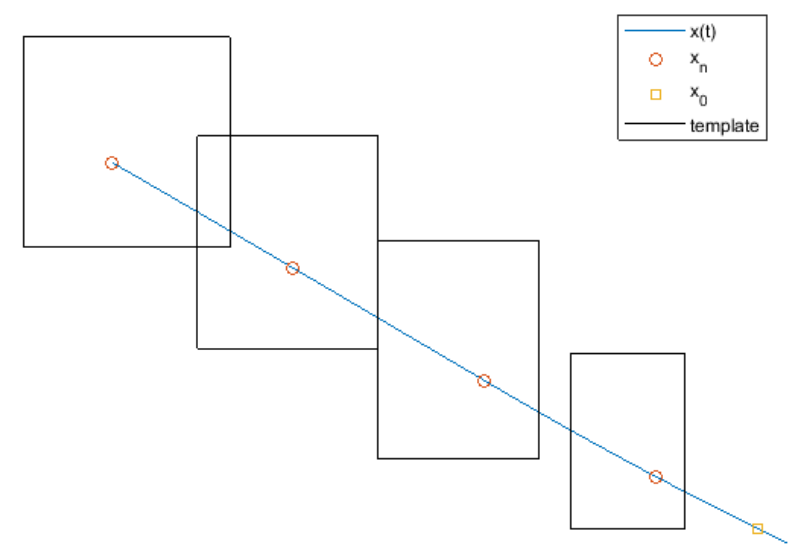

Figure 2. An example of template for $N=5$.

The proposed algorithm gives an opportunity to design full TBM trajectory tracking by extending templates with correlation gates for next scans. However, for the purpose of considerations presented in this paper, after TBD algorithm is performed (as the track initiation), the classical KF tracking is then performed. The designed extended Kalman filter uses the Newton's model with 9-element long state vector $X$. The KF parameters settling is made by filtering the plots selected at TBD stage. The further prediction is described by relations [4]:

$$
\begin{aligned}
X_{k p} & =F \cdot X_{k-1} \\
P_{k p} & =F \cdot P_{k-1} \cdot F^{T}+Q
\end{aligned}
$$

where:

$X_{k-1}$ - state vector at scan $k-1$,

$F$ - state transition matrix,

$X_{k p}$ - predicted state vector at scan $k$,

$Q$ - covariance matrix of discrete process disturbances,

$P_{k-1}$ - covariance matrix of prediction errors at scan $k-1$,

$P_{k \mathrm{p}}-$ predicted covariance matrix of filtering errors at scan $k$.

The filtering can be described as [4]: 


$$
\begin{aligned}
& u_{k p}=\left[\begin{array}{c}
\sqrt{X_{k p, 1}^{2}+X_{k p, 4}^{2}+X_{k p, 7}^{2}} \\
\operatorname{arctg}\left(\frac{X_{k p, 1}}{X_{k p, 4}}\right) \\
\operatorname{arctg}\left(\frac{X_{k p, 7}}{\sqrt{X_{k p, 1}^{2}+X_{k p, 4}^{2}}}\right)
\end{array}\right] \\
& H=\left[\begin{array}{ccccccccc}
\frac{X_{k p, 1}}{u_{k p, 1}} & 0 & 0 & \frac{X_{k p, 4}}{u_{k p, 1}} & 0 & 0 & \frac{X_{k p, 7}}{u_{k p, 1}} & 0 & 0 \\
\frac{X_{k p, 4}}{X_{k p, 1}^{2}+X_{k p, 4}^{2}} & 0 & 0 & -\frac{X_{k p, 1}}{X_{k p, 1}^{2}+X_{k p, 4}^{2}} & 0 & 0 & 0 & 0 & 0 \\
-\frac{X_{k p, 1} X_{k p, 7}}{u_{k p, 1}^{2} \sqrt{X_{k p, 1}^{2}+X_{k p, 4}^{2}}} & 0 & 0 & -\frac{X_{k p, 1} X_{k p, 7}}{u_{k p, 1}^{2} \sqrt{X_{k p, 1}^{2}+X_{k p, 4}^{2}}} & 0 & 0 & \frac{\sqrt{X_{k p, 1}^{2}+X_{k p, 4}^{2}}}{u_{k p, 1}^{2}} & 0 & 0
\end{array}\right] \\
& S=H \cdot P_{k p} \cdot H^{T}+R \\
& K_{k}=P_{k p} \cdot H^{T} \cdot S^{-1} \\
& X_{k}=X_{k p}+K_{k}\left(u_{k}-u_{k p}\right) \\
& P_{k}=\left(\mathrm{I}-K_{k} \cdot H\right) P_{k p}
\end{aligned}
$$

where:

$u_{k p}$ - predicted measurement error,

$H$ - observation model,

$S$ - matrix of innovation,

$R$ - covariance matrix of measurement errors,

$K_{k}$ - Kalman gain matrix,

$u_{k}$ - measurement error,

$I$ - identity matrix

and index $x x, n$ means the $n$-th element of vector.

Dimensions of the correlation gates are calculated based on the matrix of innovation with certain coefficients. Most of the TBM objects do not make turning maneuvers, therefore a single EKF is used.

\section{RESULTS}

Two tests have been made in order to verify the KF parameters settling. In the first one the number of the created tracks upon all the simulated trajectories has been examined in both cases: with and without using the TBD algorithm. Next, the dimensions of the correlation gates of selected track have been tested. The tracking chain has been modified by switching off the TBD initiation stage or changing the decision rule of the binary integrator.

The testing data have been generated using the simulator described in [5]. The time of the antenna revolution has been set to $10 \mathrm{~s}$, and other filter parameters have been set as follows:

- allowable objects dynamics: $10 \mathrm{~g}$,

- $\sigma_{\mathrm{R}}=220 \mathrm{~m}$, 
- $\sigma_{\text {azim }}=0.3^{\circ}$,

- $\sigma_{\text {elev }}=0.6^{\circ}$.

In the first test, 60 TBM objects moving into different directions have been simulated simultaneously with random launch point distributed in the radar range. When TBD algorithm was used, 42 tracks were initiated. The unsupported Kalman filter generated 141 tracks. This can be concluded that the TBDs do not detect all the simulated trajectories, but when they do the tracking is more reliable than classical tracking. From the tactical point of view the number of created tracks exceeding the number of objects is unacceptable.

The second test scenario included only one TBM object's trajectory moving towards the radar. The size of the correlation gates has been examined in the case of switched off TBD initiation stage or different decision threshold of $M / N$ algorithm. The object's trajectory was firstly generated without considering the measurements error. In the second stage of the test the measurement error of constant level was included in order to ensure that the results are comparable.

The dimensions of the correlation gates in the case of none measurement error in range, azimuth and elevation contain tables 1-3 respectively.

Table 1. The correlation gate dimension in range in consecutive scans for different tracking algorithms and none measurement errors.

\begin{tabular}{|l|l|l|l|l|l|}
\hline number of scan & \multicolumn{1}{|c|}{ KF } & \multicolumn{1}{|c|}{$\mathbf{3 / 5}$} & $\mathbf{4 / 5}$ & \multicolumn{1}{|c|}{$\mathbf{5 / 5}$} \\
\hline 1 & 8764.43 & & - & - & - \\
\hline 2 & 4869.30 & 4869.30 & - & - & - \\
\hline 3 & 5369.84 & 5396.84 & 5396.84 & - & - \\
\hline 4 & 5505.57 & 5505.57 & 5505.57 & 5505.57 & - \\
\hline 5 & 5542.24 & 5542.24 & 5542.24 & 5542.24 & 5542.24 \\
\hline
\end{tabular}

Table 2. The correlation gate dimension in azimuth in consecutive scans for different tracking algorithms and none measurement errors.

\begin{tabular}{|l|l|l|l|l|l|}
\hline number of scan & \multicolumn{1}{|c|}{ KF } & \multicolumn{1}{|c|}{$\mathbf{2 / 5}$} & \multicolumn{1}{c|}{$\mathbf{3 / 5}$} & $\mathbf{4 / 5}$ & $\mathbf{5 / 5}$ \\
\hline 1 & 0.09098 & & - & - & - \\
\hline 2 & 0.06583 & 0.06583 & - & - & - \\
\hline 3 & 0.07322 & 0.07322 & 0.07322 & - & - \\
\hline 4 & 0.07848 & 0.07848 & 0.07848 & 0.07848 & - \\
\hline 5 & 0.08278 & 0.08278 & 0.08278 & 0.08278 & 0.08278 \\
\hline
\end{tabular}

Table 3. The correlation gate dimension in elevation in consecutive scans for different tracking algorithms and none measurement errors.

\begin{tabular}{|l|l|l|l|l|l|}
\hline number of scan & \multicolumn{1}{|c|}{$\mathbf{K F}$} & \multicolumn{1}{|c|}{$\mathbf{2 / 5}$} & $\mathbf{3 / 5}$ & $\mathbf{4 / 5}$ & \multicolumn{1}{|c|}{$\mathbf{5 / 5}$} \\
\hline 1 & 0.11731 & & - & - & \\
\hline 2 & 0.11223 & 0.11223 & - & - & - \\
\hline 3 & 0.10555 & 0.10555 & 0.10555 & - & - \\
\hline 4 & 0.11456 & 0.11456 & 0.11456 & 0.11456 & - \\
\hline 5 & 0.11885 & 0.11885 & 0.11885 & 0.11885 & 0.11885 \\
\hline
\end{tabular}


The obtained results showed that in the case of no measurement errors the TBD algorithm have no influence on tracking quality. The only difference is the delayed start of work of KF in reference to used decision rule in the TBD module. Similar conclusions can be drawn from the analysis of the tracking visualization presented in Figure 3. On the left the result of using the Kalman filter only tracking is shown, while on the right one may observe the tracking with the TBD initialization algorithms with $3 / 5$ decision rule.

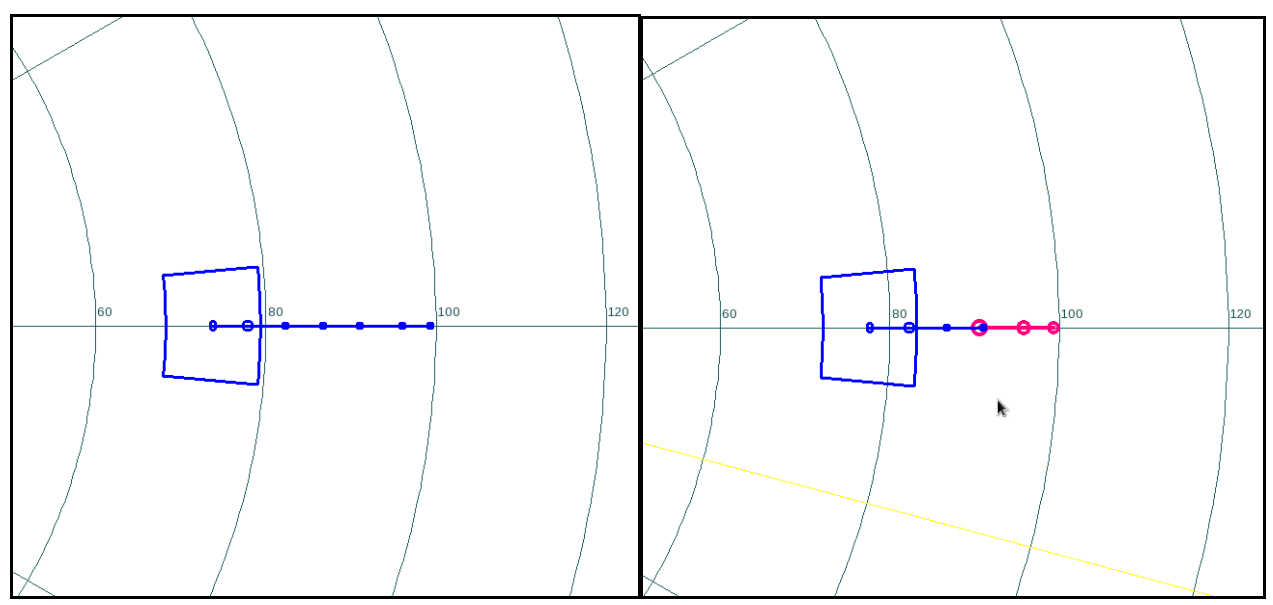

Figure 3. A visualization of tracking of simulated TBM trajectory without measurement error when only KF is used and with track initiation by TBD with decision rule set to $3 / 5$.

Similarly, the correlation gates dimensions in range, azimuth and elevation in the case of considering the radar measurement errors contain tables 4-6.

Table 4. The correlation gate dimension in range in consecutive scans for different tracking algorithms considering measurement errors.

\begin{tabular}{|l|l|l|l|l|l|}
\hline number of scan & \multicolumn{1}{|c|}{$\mathbf{K F}$} & \multicolumn{1}{|c|}{$\mathbf{2 / 5}$} & $\mathbf{4 / 5}$ & $\mathbf{5 / 5}$ \\
\hline 1 & & & & - & \\
\hline 2 & - & 5039.44 & - & - & - \\
\hline 3 & - & 15000.00 & 5340.34 & - & - \\
\hline 4 & - & 9079.47 & 5541.38 & 5541.38 & - \\
\hline 5 & - & 15000.00 & 5610.82 & 5610.82 & 5610.82 \\
\hline
\end{tabular}

Table 5. The correlation gate dimension in azimuth in consecutive scans for different tracking algorithms considering measurement errors.

\begin{tabular}{|l|l|l|l|l|l|}
\hline number of scan & \multicolumn{1}{|c|}{$\mathbf{2 / 5}$} & \multicolumn{1}{|c|}{$\mathbf{3 / 5}$} & \multicolumn{1}{|c|}{$\mathbf{4 / 5}$} & \multicolumn{1}{|c|}{$\mathbf{5 / 5}$} \\
\hline 1 & & & & - & \\
\hline 2 & - & 0.06614 & - & - & - \\
\hline 3 & - & 0.24435 & 0.07096 & - & - \\
\hline 4 & - & 0.10299 & 0.08077 & 0.08077 & - \\
\hline 5 & - & 0.24435 & 0.08245 & 0.08245 & 0.08245 \\
\hline
\end{tabular}


Table 6. The correlation gate dimension in elevation in consecutive scans for different tracking algorithms considering measurement errors.

\begin{tabular}{|l|l|l|l|l|l|}
\hline number of scan & \multicolumn{1}{|c|}{$\mathbf{2 / 5}$} & \multicolumn{1}{|c|}{$\mathbf{3 / 5}$} & $\mathbf{4 / 5}$ & $\mathbf{5 / 5}$ \\
\hline 1 & - & & - & - & - \\
\hline 2 & - & 0.11042 & - & - & - \\
\hline 3 & - & 0.33812 & 0.10455 & - & - \\
\hline 4 & - & 0.12184 & 0.11783 & 0.11783 & 0.11622 \\
\hline 5 & - & 0.33135 & 0.11622 & 0.11622 & \\
\hline
\end{tabular}

If measurement errors are considered, the algorithms with decision rule at least $3 / 5$ ensure the tracking settling. In the case of using only KF, many tracks are created, and they cannot be compared with TBD results. As shown in Figure 4, the measurement errors cause the serious problem with settling of KF parameters and unstable tracking. In contrast, TBD algorithm initiated only one track and ensured further stable tracking by KF.

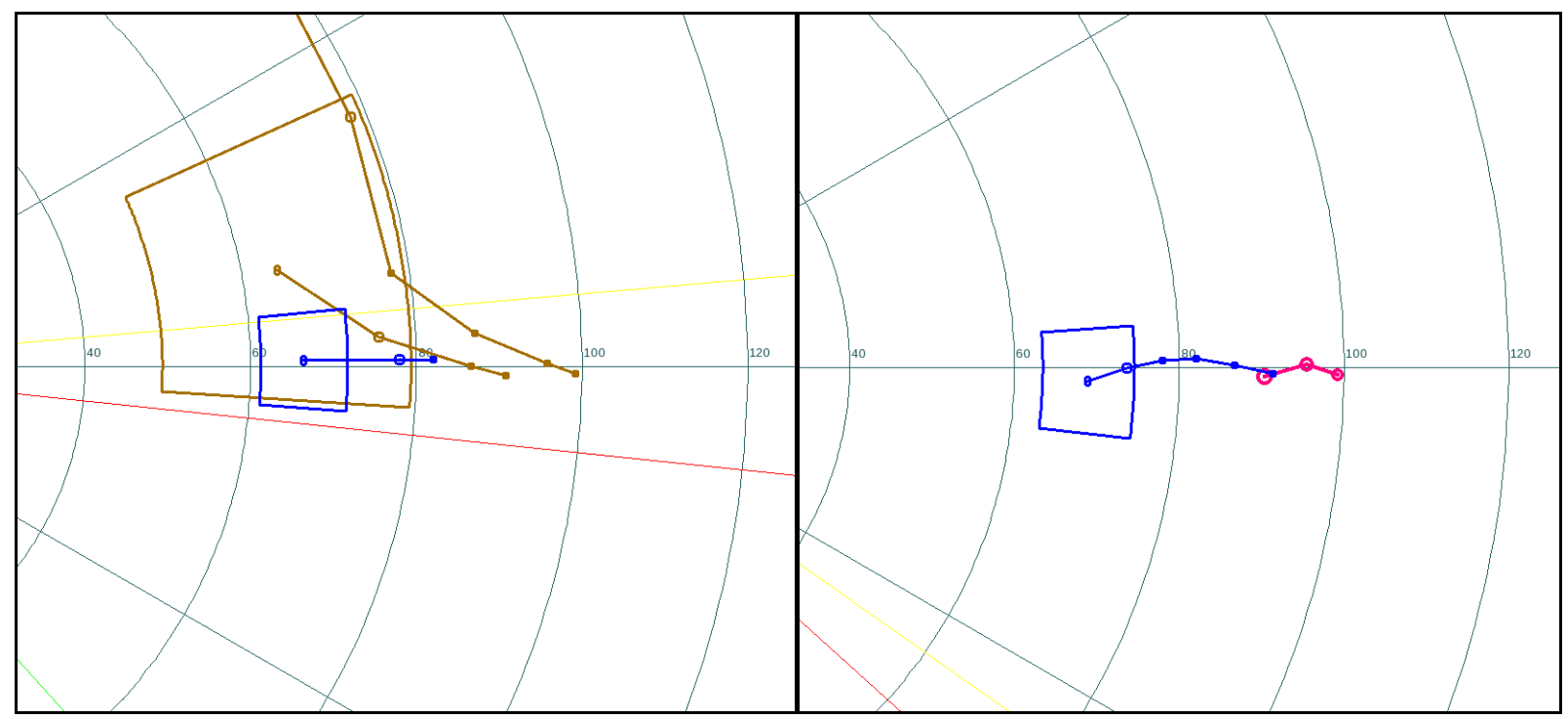

Figure 4. A visualization of tracking for simulated trajectory with included measurement errors in the case of using only KF and preprocessed by TBD with $3 / 5$ decision rule.

Also the case of tracking when the decision rule is set to 2/5 is very interesting. Their following stage are presented in Figure 5. 


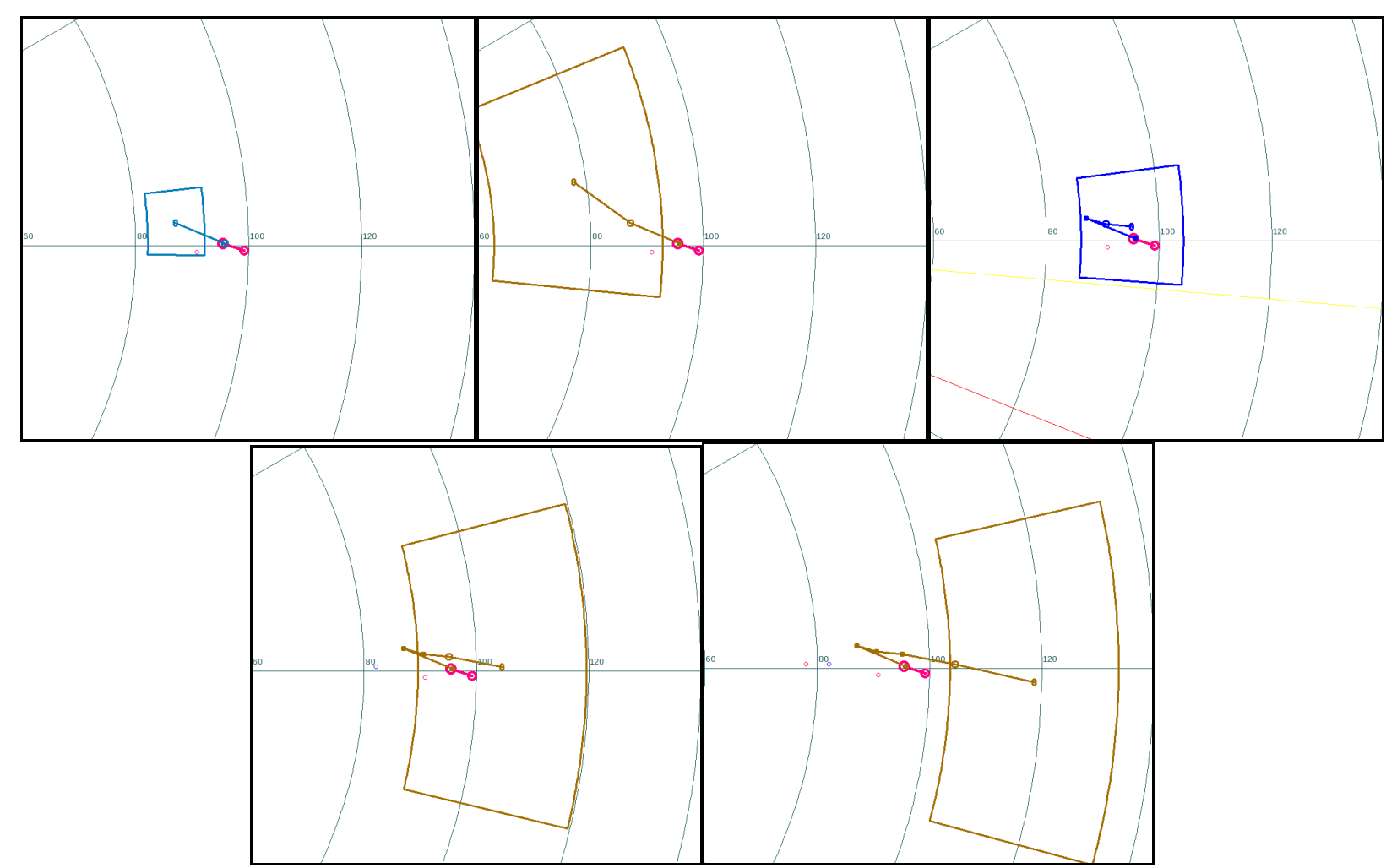

Figure 5. A visualization of tracking for simulated trajectory with included measurement errors in the case of using TBD algorithms with $2 / 5$ decision rule.

In this case the TBD algorithm detected first two plots of trajectory and computed the KF correlation gate. Although the third plot has seemed to fit the gate, in fact the elevation condition has not been met. The gate widened up to the assumed maximum size containing the next plot, and the track has been updated. Unfortunately, the filter parameters settled based on this plot set have effected in object's turning back. That was partially caused by a relatively small object-to-radar distance. Then, the following stages of tracking have not been proceeded properly, and in the consequence the track has been deleted.

\section{SUMMARY}

In this paper the influence of TBD algorithm on the next steps of TBM objects tracking by Kalman filter has been examined. The presented results showed that such configuration of the tracking chain allows to initiate smaller number of false tracks. In the same time, if measurement errors are considered, the use of the TBD algorithm ensures more reliable tracking than pure Kalman filter. Further tests of settling Kalman filter parameters by TBD algorithm should take into consideration the occurrence of false alarms in the neighborhood of the simulated track.

\section{REFERENCES}

[1] Blackamn S. and Popoli R.. [Design and Analysis of Modern Tracking Systems]. Artech House. Norwood. (1999)

[2] Grewal M.S. and. Andrews A.P.. [Kalman Filtering. Theory and Practice Using MATLAB]. John Willey \& Sons. (2001)

[3] Sankowski M.. Uruski P. and Blok E.. "Application of Track-Before-Detect Algorithm to Detection of Ballistic Missiles in Air-Defense Radar." 15th Int. Conf. on Mic.. Radar and Wireless Communications. 97-100 (2004).

[4] Kaniewski P.. Smagowski P. and Konatowski S. "Ballistic Target Tracking with Use of Cinetheodolites." International Journal of Aerospace Engineering. 2019(3240898). (2019).

[5] Wajszczyk B.. Motyl K. and Sienicki K.. "The concept of the radar environment simulator software." Proc. SPIE 10715. 107150G (2018). 